\title{
SQSTM1 Mutations and Glaucoma
}

\author{
Todd E. Scheetz ${ }^{1,2}$, Ben R. Roos ${ }^{1,2}$, Frances Solivan-Timpe ${ }^{1,2}$, Kathy Miller, , Adam \\ P. DeLuca ${ }^{1,2}$, Edwin M. Stone ${ }^{1,2}$, Young H. Kwon ${ }^{1,2}$, Wallace L. M. Alward ${ }^{1,2}$, Kai Wang ${ }^{2,3}$, \\ John H. Fingert ${ }^{1,2 *}$
}

1 Department of Ophthalmology and Visual Sciences, Carver College of Medicine, University of lowa, lowa City, lowa, United States of America, 2 Stephen A. Wynn Institute for Vision Research, University of lowa, lowa City, lowa, United States of America, 3 Department of Biostatistics, College of Public Health, University of lowa, lowa City, lowa, United States of America

* john-fingert@uiowa.edu

\section{Abstract}

Glaucoma is the most common cause of irreversible blindness worldwide. One subset of glaucoma, normal tension glaucoma (NTG) occurs in the absence of high intraocular pressure. Mutations in two genes, optineurin (OPTN) and TANK binding kinase 1 (TBK1), cause familial NTG and have known roles in the catabolic cellular process autophagy. TKB1 encodes a kinase that phosphorylates OPTN, an autophagy receptor, which ultimately activates autophagy. The sequestosome (SQSTM1) gene also encodes an autophagy receptor and also is a target of TBK1 phosphorylation. Consequently, we hypothesized that mutations in SQSTM1 may also cause NTG. We tested this hypothesis by searching for glaucoma-causing mutations in a cohort of NTG patients $(n=308)$ and matched controls $(n=$ 157) using Sanger sequencing. An additional 1098 population control samples were also analyzed using whole exome sequencing. A total of 17 non-synonymous mutations were detected which were not significantly skewed between cases and controls when analyzed separately, or as a group $(p>0.05)$. These data suggest that SQSTM1 mutations are not a common cause of NTG.

\section{Introduction}

Glaucoma is an important public health problem that is the common cause of irreversible blindness worldwide [1]. Glaucoma is a disease of the optic nerve, which carries visual signals from the retina in the eye to the brain. The key features of glaucoma are damage to the optic nerve, which is visible on ophthalmic exam, and a characteristic pattern of visual field loss. The most common type of glaucoma in many nations is primary open angle glaucoma (POAG), which occurs in the absence of any other ocular abnormalities including anatomical defects that might obstruct fluid egress from the eye. High intraocular pressure (IOP) is a potent risk factor for glaucoma [2]. The higher the IOP, the greater the risk for developing glaucoma, however, glaucoma can occur at any IOP [3]. When glaucoma occurs below an arbitrary threshold pressure, $21 \mathrm{~mm} \mathrm{Hg}$, it is frequently termed normal tension glaucoma (NTG). 
The basic causes of NTG have been explored with genetic studies. Population-based studies using genome-wide association studies have identified several risk factors that contribute to the development of complex genetic forms of NTG, including S1 RNA binding domain 1 (SRBD1) [4], elongation of long chain fatty acids family member 5 (ELOVL5) [4], toll-like receptor 4 (TLR4) [5], and CDKN2B-AS1 [6,7]. These NTG risk factors are present in NTG patients at a statistically higher frequency than they are observed in individuals with healthy eyes [8].

Genetic studies have also been conducted on large families with numerous members diagnosed with NTG. Linkage analysis of one such pedigree identified optineurin $(O P T N)$ as an NTG-causing gene. One mutation in OPTN, E50K, was subsequently associated with $1-2 \%$ of cases of NTG [9-11]. More recently, study of another large NTG pedigree showed that copy number variation (duplication) of the TANK binding kinase 1 (TBK1) gene is another cause of NTG [12]. Population studies later demonstrated that copy number variations in TBK1 (duplications, triplications, and one deletion) are associated with approximately $1 \%$ of NTG cases [12-16]. The E50K OPTN mutation and TBK1 copy number variations are high penetrance mutations. Individuals with either an OPTN mutation or a TBK1 mutation almost always develop NTG, while these mutations are only rarely observed in normal individuals [8].

\section{NTG genes and autophagy}

Both NTG genes (TBK1 and OPTN) have important roles in autophagy suggesting that macroautophagy may be a key mechanism in NTG. Macroautophagy, hereafter referred to as autophagy, is an ancient catabolic cellular process that digests intracellular structures for energy in times of nutritional deprivation [17]. The same mechanisms are also used by cells to eliminate accumulating proteins or intracellular pathogens [17-19]. In autophagy a double membrane endosome, the autophagosome, forms around targeted materials within the cytoplasm. The autophagosome fuses with the lysosome and the contents of the resulting autophagolysosome are degraded. Decreased levels of autophagy have been associated with neurodegenerative diseases including Parkinson disease, Huntington disease, and amyotrophic lateral sclerosis (ALS) with the presumption that reduced ability to eliminate accumulating materials in neurons leads to their death and disease [20-24]. Alternatively, increased autophagy has been observed in animal models of glaucoma and optic nerve disease [25,26]. In this case, excess catabolic activity from autophagy may destroy cellular components necessary for survival [25]. Consequently, defects in TBK1 or OPTN and autophagy are a plausible cause of the retinal ganglion cell death and optic nerve damage that are central features of glaucoma.

TBK1 encodes a kinase that stimulates autophagy by phosphorylating OPTN at Ser177 [18]. Phosphorylation of OPTN activates its functional domains to link structures targeted for elimination with proteins of an assembling autophagosome. Specifically OPTN's ubiquitin binding domain associates with ubiquitinated targets of autophagy and its LC3 binding domain associates with a key component of the forming autophagosome, i.e. microtubule-associated protein light chain 3 (LC3). In this way OPTN acts as an autophagy receptor, linking targets of autophagy with specific elements of the autophagosome and promotes autophagy [18].

There is evidence that NTG-causing mutations in OPTN and TBK1 promote increased activation of autophagy. Yeast two-hybrid studies indicate that an NTG-causing OPTN mutation, enhances the association between OPTN and TBK1 [27], which would likely increase the opportunity for TBK1 to phosphorylate OPTN and stimulate autophagy. Also, all but one [15] of the TBK1 CNVs discovered in NTG patients to date are duplications and triplications, which would similarly favor activation of OPTN and autophagy. Finally, we have studied autophagy in retinal ganglion cell-like neurons that were generated using skin biopsies collected from patients with NTG caused by a TBK1 duplication using induced pluripotent stem 
cell methods. Key markers of autophagy are increased in retinal ganglion cell-like neurons with TBK1 gene duplications [28].

The discovery that two autophagy genes cause NTG suggests that additional autophagy genes may also be important in glaucoma. One autophagy gene, sequestosome 1 (SQSTM1) or p62, is an especially good candidate for causing NTG. There are similarities with OPTN that suggest SQSTM1 may also be an NTG gene. Both OPTN and SQSTM1 encode proteins that are phosphorylated by TBK1 and function as autophagy receptors [18,29]. Moreover, the genes that cause ALS overlap with the genes that cause NTG. Previous studies have shown that mutations in TBK1 [30,31], OPTN [32] and SQSTM1 [33,34] are associated with ALS, suggesting a general link between the genetics of ALS and NTG. These data suggest that while some SQSTM1 mutations are associated with ALS, other mutations may cause NTG. Consequently, we investigated SQSTM1 for glaucoma-causing mutations in a cohort of NTG patients.

\section{Materials and Methods Patient Cohorts}

Subjects enrolled in the study provided written informed consent and the research was approved by the University of Iowa's Internal Review Board. A cohort of 308 patients with NTG had excavation of their optic nerve head with resultant glaucomatous visual field loss in at least one eye. Glaucomatous optic nerves had cup-to-disc ratios of greater than 0.7 with thinning of the neural rim, asymmetry of the optic nerve cup-to- disc ratio greater than 0.2 , or progressive loss of the neural rim identified with baseline disc photography. Patients were 40 years of age or older at diagnosis and had gonioscopically open iridocorneal angles (angle greater than Shaffer grade II). Enrollment criteria included a maximum IOP of $21 \mathrm{mmHg}$ or less. A cohort of 157 control subjects over 50 years of age were examined by board-certified ophthalmologists and were judged to have normal optic nerve head appearance and of IOP $21 \mathrm{~mm} \mathrm{Hg}$ or less. An additional cohort of 1098 patients enrolled in an inherited eye disease study at the University of Iowa were used as a population control cohort. These patients have ocular diagnoses unrelated to glaucoma ( $90 \%$ have photoreceptor degenerations or macular dystrophies).

\section{DNA sequencing}

The entire coding sequence of SQSTM1 was PCR amplified from DNA samples collected from NTG patients $(n=308)$ and normal control subjects $(n=157)$ using overlapping primer pairs in standard PCR reactions (primers listed in Table 1). Amplified DNA sequences were determined using BigDye chemistry and a 3730 automated sequencer (Applied Biosciences, Foster City, CA) as previously described [12]. The coding sequence of SQSTM1 was determined from

Table 1. SQSTM1 primer sequences.

\begin{tabular}{lll}
\hline & Forward Primer & Reverse Primer \\
\cline { 2 - 3 } Exon 1 & GGAAGGGAGAGTAGTGAAGG & CTTGGTCACCACTCCAGTCA \\
Exon 2 & AGCCTGTGAGTGTCCTTT & ACAGCCCTCAAATTGCTGAC \\
Exon 3 / 4 & GCAGTGACAGCCCCACAGT & GGCTGCCTGACTACTGTCAC \\
Exon 5 & GACCTTGGCAAGAAGGTGAC & CAGTATTCCAGGTGAAAGTTACATAAA \\
Exon 6 & CTTGCAGGTGCATCCTTGG & GTGCAGGCCACAGATCACTA \\
Exon 7 & CCTAGACCCCTGCAGCCTTA & AGGGCAGGATGCTCTAAAGG \\
Exon 8 & GGGTATGTGTTTCGGTCAC & TCCTGGAAGAAGGAGAGAA \\
\hline
\end{tabular}

These primer sequences were used both for PCR amplification and for Sanger sequencing of the SQSTM1 coding sequences.

doi:10.1371/journal.pone.0156001.t001 
DNA samples collected from a cohort of patients with retinal degenerations $(\mathrm{n}=1098)$ using whole exome sequencing with the Agilent v5 kit (Santa Clara, CA) and the Illumina HiSeq2000/2500 (San Diego, CA) and subjected to quality control metrics as previously described [35]. The reads were aligned to the genome using BWA [36], and resulting variants were called using the genome analysis toolkit (GATK) [37]. Variants were annotated and prioritized for follow-up using methods we have described previously [38]. Briefly, variants were first filtered to retain those predicted to alter coding sequence or impact a splice site based upon the RefSeq transcript (NM_003900.4). All variants detected in the SQSTM1 gene by exome sequencing of this Iowa Population Control cohort are listed in S1 Table. Variants that were seen in at least $1 \%$ in any ExAC population [39] were assumed to be too common to cause disease and were removed.

\section{Statistics}

Power calculations were conducted to assess our ability to detect SQSTM1 mutations. Given the cohort sizes, we had $80 \%$ power to detect a statistically significant skew in non-synonymous variants if they occurred at a frequency of $3.5 \%$ or greater in the NTG population $(\mathrm{n}=308)$ than in the control population $(\mathrm{n}=157)$. Similarly, this study had $80 \%$ power to detect a statistically significant skew in non-synonymous variations if they occurred at a frequency $1.2 \%$ or greater in the NTG population $(n=308)$ than in the population control group $(n=1098)$. The frequency of non-synonymous variants were compared using Fisher's exact test with a threshold of 0.05 for significance.

\section{Results}

A cohort of 308 NTG patients and two control cohorts (157 normal controls and 1098 population controls) were tested for glaucoma-causing mutations in the coding sequences of the SQSTM1 gene using automated Sanger DNA sequencing. Demographic features of these patients are shown in Table 2. A total of 27 unique sequence variations were detected and all were in Hardy-Weinberg equilibrium. Of these variations, 17 were non-synonymous coding sequence mutations (Table 3) and 10 were synonymous changes (Table 4). Two rare non-synonymous mutations (Pro29Ser and Arg212Cys) were detected in the NTG cohort but were

Table 2. Patient and control cohort demographics.

\begin{tabular}{|c|c|c|c|}
\hline & lowa NTG Cohort & lowa Normal Controls & lowa Population Controls \\
\hline Total & 308 & 157 & 1098 \\
\hline Female & 213 & 84 & 589 \\
\hline Male & 94 & 73 & 507 \\
\hline$X X Y$ & 0 & 0 & 2 \\
\hline Unknown & 1 & 0 & 0 \\
\hline Mean age at enrollment & 69.4 & 70.1 & NA \\
\hline \multicolumn{4}{|l|}{ Diagnosis } \\
\hline NTG & 308 & 0 & NA \\
\hline Photoreceptor degeneration & NA & NA & 834 \\
\hline Maculopathy & NA & NA & 148 \\
\hline Congenital cataract & NA & NA & 77 \\
\hline Other & NA & NA & 39 \\
\hline
\end{tabular}

Abbreviations: not available (NA).

doi:10.1371/journal.pone.0156001.t002 


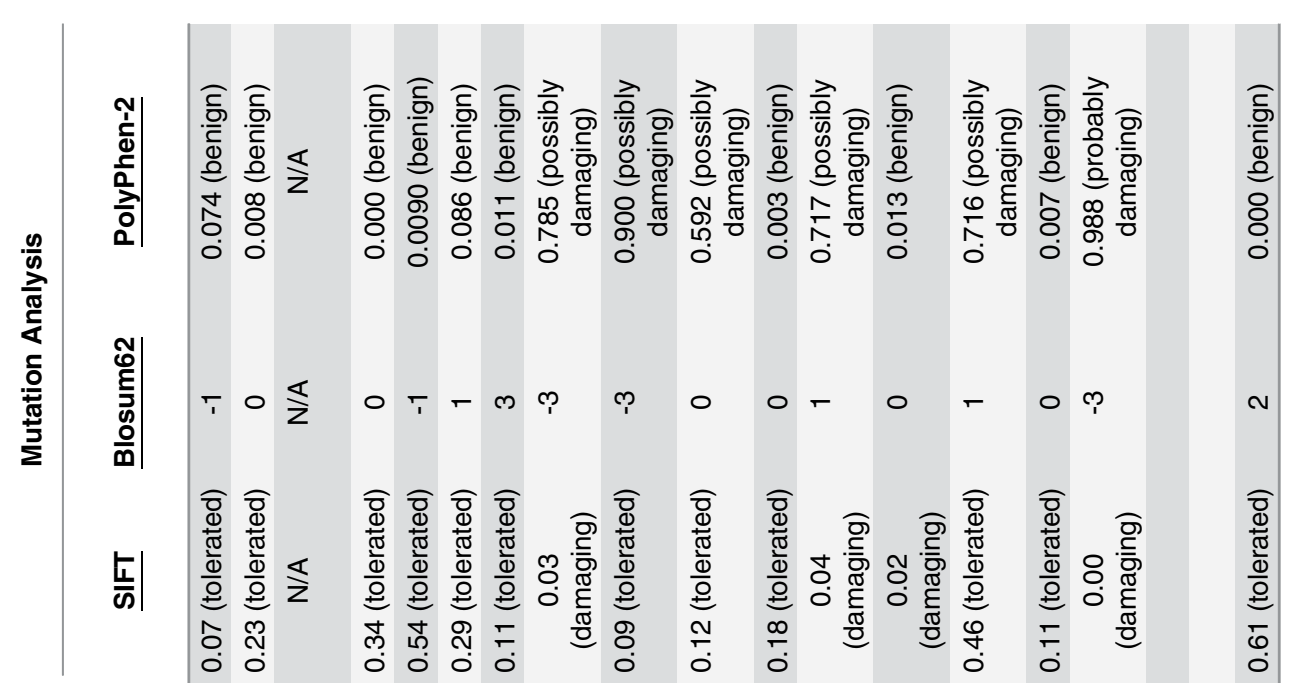

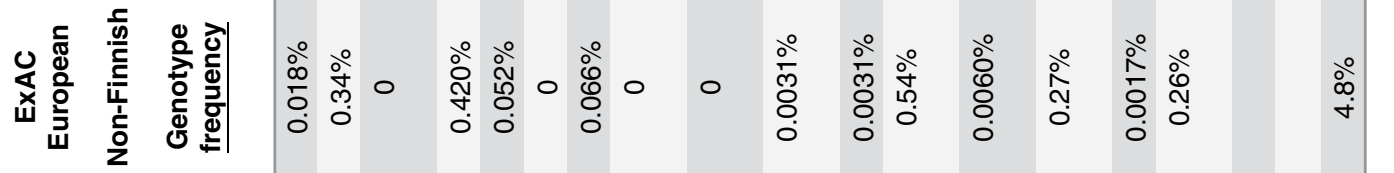

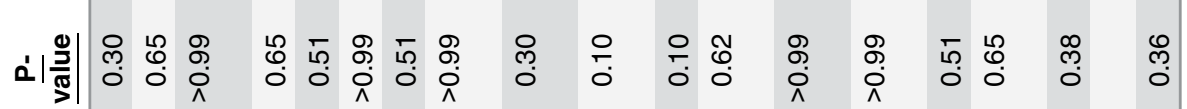

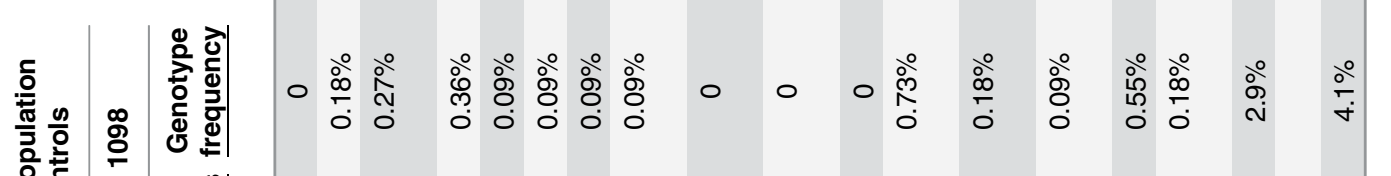

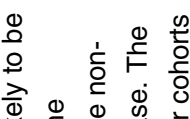

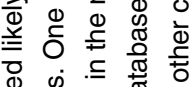

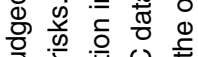

.

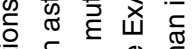

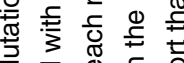

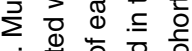

里

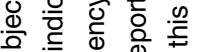

के

인

ठํ워

당

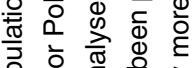

ํํㅇำ

을 है 은

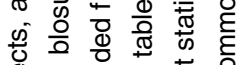

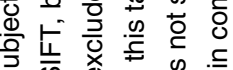

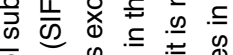

인 क

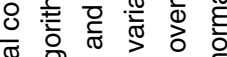

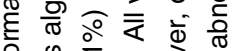

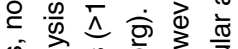

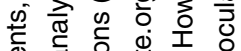

ब

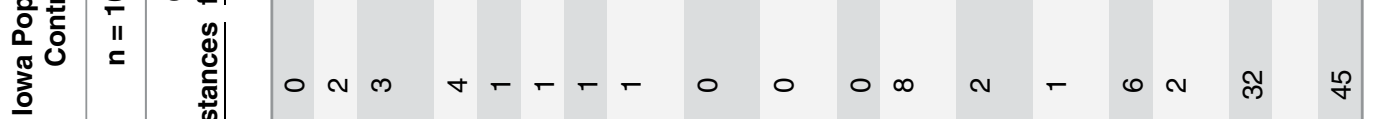

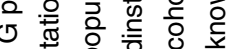

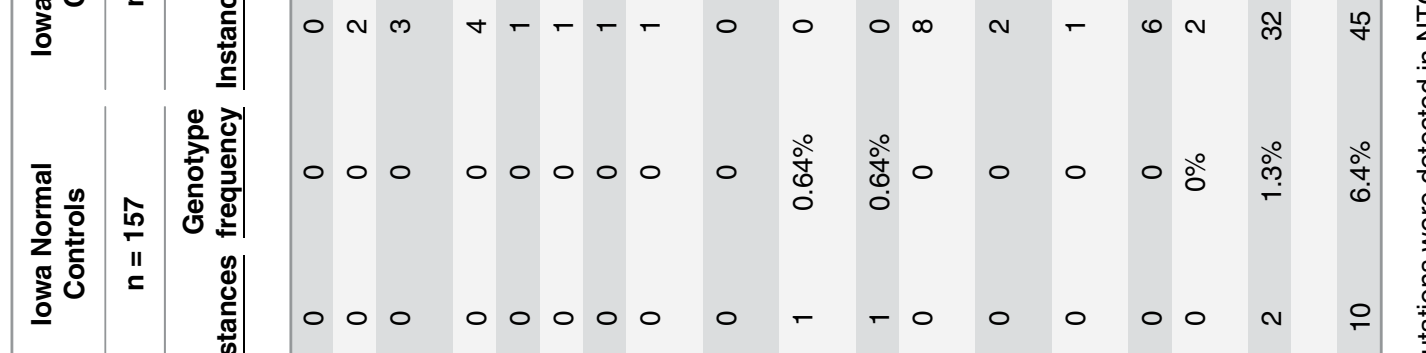

$z$ दे 응 잉 일

$\leqq$ ष

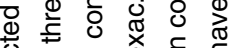

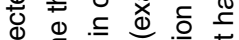

흥 춯웡

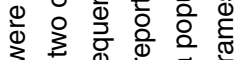

की

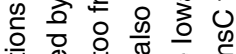

贾

द

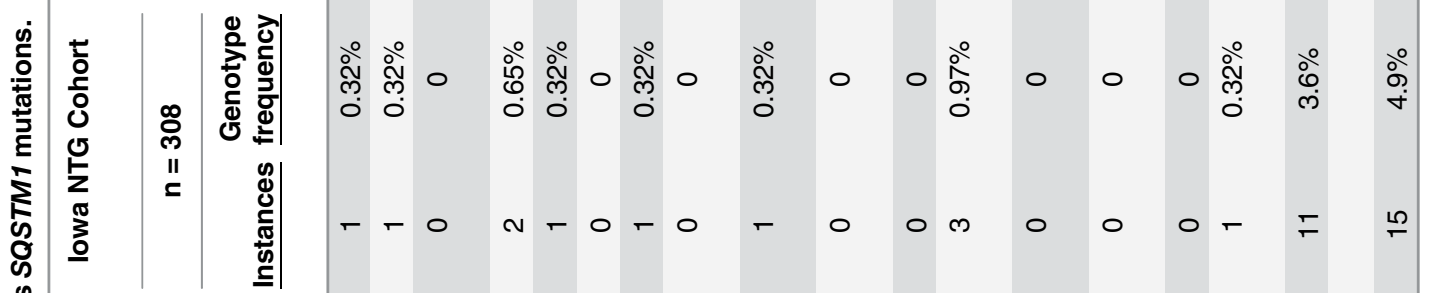

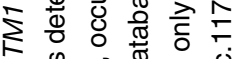
क \& क tᄒ 青 次 승 为

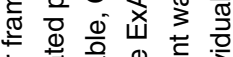

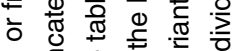
号

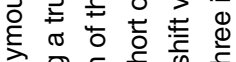


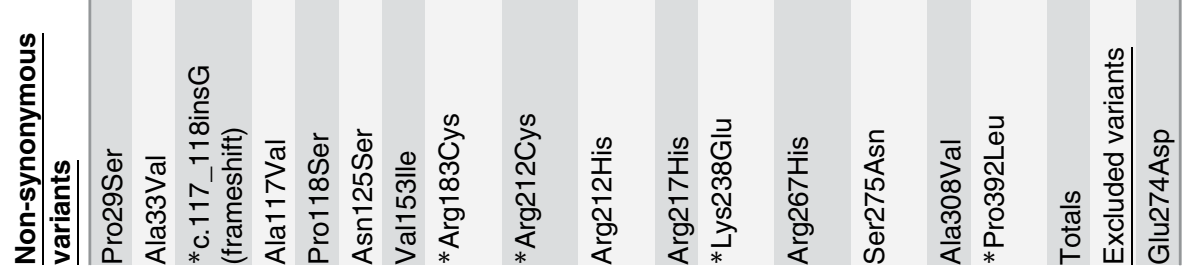

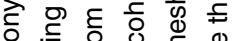

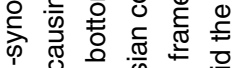

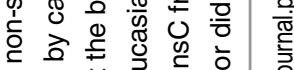


Table 4. Synonymous SQSTM1 mutations.

\begin{tabular}{|c|c|c|c|c|c|c|c|}
\hline \multirow[b]{3}{*}{ Synonymous Variants } & \multirow{2}{*}{\multicolumn{2}{|c|}{$\begin{array}{c}\text { lowa NTG cohort } \\
n=308\end{array}$}} & \multirow{2}{*}{\multicolumn{2}{|c|}{$\begin{array}{c}\text { lowa Normal Controls } \\
\qquad n=157\end{array}$}} & \multirow{2}{*}{\multicolumn{2}{|c|}{$\begin{array}{c}\text { lowa Population Controls } \\
n=1098\end{array}$}} & \multirow{3}{*}{$\begin{array}{l}\text { ExAC European } \\
\text { Non-Finnish cohort } \\
\text { Allele Frequency }\end{array}$} \\
\hline & & & & & & & \\
\hline & Instances & Allele Frequency & Instances & Allele Frequency & Instances & Allele Frequency & \\
\hline Gly61Gly & 1 & $0.16 \%$ & 0 & $0 \%$ & 9* & $0.41 \%$ & - \\
\hline Ser180Ser & 1 & $0.16 \%$ & 0 & $0 \%$ & 0 & $0 \%$ & $0.0092 \%$ \\
\hline Asp292Asp & 327 & $53.1 \%$ & 161 & $51 \%$ & 1185 & $53.90 \%$ & $62 \%$ \\
\hline Gly302Gly & 0 & $0 \%$ & 1 & $0.32 \%$ & 4 & $0.18 \%$ & $0.026 \%$ \\
\hline Ala308Ala & 0 & $0 \%$ & 1 & $0.32 \%$ & 2 & $0.09 \%$ & $0.069 \%$ \\
\hline Arg312Arg & 327 & $53.1 \%$ & 162 & $52 \%$ & 1144 & $52 \%$ & $56 \%$ \\
\hline Ser318Ser & 16 & $2.6 \%$ & 9 & $2.9 \%$ & 56 & $2.50 \%$ & $3.1 \%$ \\
\hline Ser328Ser & 2 & $0.32 \%$ & 2 & $0.64 \%$ & 10 & $0.45 \%$ & $0.55 \%$ \\
\hline Pro348Pro & 1 & $0.16 \%$ & 1 & $0.32 \%$ & 19 & $0.86 \%$ & $0.040 \%$ \\
\hline Pro392Pro & 1 & $0.16 \%$ & 0 & $0 \%$ & 1 & $0.05 \%$ & $0.0075 \%$ \\
\hline
\end{tabular}

Ten synonymous SQSTM1 mutations were detected in NTG patients, normal control subjects, and population control subjects. The frequency of each mutation in the non-Finnish Caucasian cohort of the ExAC public database is also reported. All variants in this table have been previously reported in the ExAC database

* DNA sequencing results for this variant were reported for 1092 of 1098 of the lowa Population.

doi:10.1371/journal.pone.0156001.t004

absent from both control cohorts. Similarly, eight rare non-synonymous variants were detected only in the control cohorts. A single conservative variant, Glu274Asp, was detected too frequently in NTG patients (4.9\%) and in control subjects (6.4\% in normal controls and $4.1 \%$ in population controls) to be a cause of glaucoma and was excluded from analysis. Non-synonymous variants in SQSTM1 were detected at a higher frequency in the NTG cohort (3.6\%) than in the control cohorts (1.3\% in normal controls and $2.9 \%$ in population controls). The frequency of SQSTM1 mutations was 2 to 3-fold higher in NTG patients than in Iowa normal controls, however, this difference in frequency was not statistically significant $(\mathrm{p}=0.23)$. Finally, no statistically significant difference in the frequency of non-synonymous SQSTM1 mutations was detected when all three cohorts (Iowa NTG, Iowa normal controls, and Iowa population controls) were compared $(\mathrm{p}=0.38)$

We also analyzed the predicted deleterious effects of the 17 mutations detected in the SQSTM1 gene using three algorithms, SIFT, blosum62, and PolyPhen (Table 3). Twelve of the mutations were judged to be benign by at least two of the three algorithms, while four missense mutations were judged damaging by at least two of the three algorithms (Arg183Cys, Arg212Cys, Lys238Glu, and Pro392His). These four possibly damaging missense mutations were all present in patient and control subjects at similar frequencies. A fifth mutation, c.117_118insG(frameshift), was also judged to be likely detrimental as it leads to a series of 29 aberrant amino amino acids followed by a premature termination of the encoded protein after the $69^{\text {th }}$ amino acid. The c.117_118insG mutation was detected in $3(0.27 \%)$ of 1098 population control subjects and was not identified in NTG patients. Overall, there was no skew in the frequency of the frameshift and four missense mutations judged to be potentially damaging between NTG patients and control cohorts $(\mathrm{p}=0.31)$.

\section{Discussion}

Recently, studies of glaucoma have identified disease-causing genes (OPTN and TBK1) that directly interact with each other in the same biological pathway. TBK1 encodes a kinase that 
phosphorylates OPTN and stimulates autophagy and mutations in TBK1 or OPTN appear to cause glaucoma via dysregulation of autophagy. Consequently, it was plausible to search for glaucoma-causing mutations in other autophagy genes. In addition to OPTN, TBK1 phosphorylates two other autophagy receptor proteins, SQSTM1 and calcium binding and coiled-coil domain 2 (CACOCO2). In this report we investigated the possible role of mutations in SQSTM1 in NTG pathogenesis by testing a large cohort of NTG patients for glaucoma-causing mutations. We identified SQSTM1 missense mutations in 11 (3.6\%) of 308 NTG patients and in $2(1.3 \%)$ of 157 normal controls, and in $32(2.9 \%)$ of 1098 population controls.

Statistical analysis of the SQSTM1 data identified no difference between the frequency of mutations in NTG patients and controls. Although the frequency of SQSTM1 mutations in NTG patients was slightly higher in NTG patients, the difference was not statistically significant. These data suggest that SQSTM1 mutations are not a common cause of NTG. However, it does remain possible that some SQSTM1 mutations do cause NTG. For example, the Arg212Cys mutation was detected in one NTG patient and was absent from both control populations. Moreover, all three mutation analyses (SIFT, Blosum62, and PolyPhen-2) suggested that the Arg212Cys mutation may be pathogenic. Together these data raise the possibility that this specific mutation may cause disease. However, a much larger cohort would be required to detect disease-causing mutations with statistical significance. For example, a study of 5,000 NTG patients and 5,000 controls would have $76 \%$ power to detect a statistically significant difference between a $4 \%$ frequency of SQSTM1 mutations in cases and a 3\% frequency in controls. An alternative way to support a link between mutations in SQSTM1 and NTG would be to compare inheritance of the detected mutations with the inheritance of glaucoma in family members. Unfortunately, this study utilized a cohort of unrelated NTG patients and additional family members are not available for study. Finally, the ultimate approach to investigate pathogenicity would be to generate a transgenic animals with rare SQSTM1 variants to see if they develop glaucoma.

Mutations in the SQSTM1 were previously associated with ALS. Interestingly, three of the same variants reported in ALS patients (Ala33Val, Val153Ile, and Pro392Leu) [33,34,40-45] were also detected in our population control cohort and are present at a similar rate in the ExAC database (Table 3). The presence of these variants in control subjects not known to have ALS might partially undermine their proposed role in the pathogenesis of this neurodegenerative disease.

There are potential limitations to our study. One method (Sanger Sequencing) was used to analyze the DNA of the NTG patients and the normal control subjects, while another method (whole exome sequencing) was used to analyze the DNA of the Iowa Population Control subjects. It is possible that these differences in methodology could bias to our results with different mutation detection efficiencies. However, we examined the whole exome sequencing data and found that there was an average of $88 \mathrm{X}$ coverage across the coding sequence of this gene in all subjects, which suggests that there was no bias against mutation discovery due to whole exome coverage. Also, both Sanger sequencing and whole exome sequencing detected non-synonymous mutations at similar frequencies (Table 4), suggesting that bias due to different sequencing methods is less likely. Our investigations of the SQSTM1 gene were limited to coding sequences. It is possible that mutations involving non-coding, regulatory regions of the SQSTM1 gene may be associated with NTG and went undetected by this report.

Future studies of larger cohorts with more extensive mutation detection strategies (i.e. assessing both coding and regulatory sequences) might have the power to identify rare glaucoma-causing SQSTM1 variants. Alternatively, future association studies might also have the potential to identify common SQSTM1 risk alleles for NTG. However, at present, the sum of our data suggests that coding sequence SQSTM1 mutations are not associated with NTG. 


\section{Supporting Information}

S1 Table. Variations detected in the SQSTM1 gene in control subjects by exome analysis. Column 1 (Variants detected in the SQSTM1 gene by the exome study) lists detected variants annotated relative to the hg19 reference genome. Column 2 (Variant location relative to SQSTM1 coding sequence) lists variants annotated relative to the transcript in HGVS standard nomenclature (http://varnomen.hgvs.org).

(XLSX)

\section{Author Contributions}

Conceived and designed the experiments: TES KW JHF. Performed the experiments: TES BRR FST KM APD KW. Analyzed the data: TES APD KW JHF. Contributed reagents/materials/ analysis tools: TES APD EMS YHK WLMA JHF. Wrote the paper: TES KW JHF.

\section{References}

1. Quigley HA, Broman AT. The number of people with glaucoma worldwide in 2010 and 2020. $\mathrm{Br} \mathrm{J}$ Ophthalmol. BMJ Publishing Group Ltd; 2006; 90: 262-267. doi: 10.1136/bjo.2005.081224

2. Gordon MO, Beiser JA, Brandt JD, Heuer DK, Higginbotham EJ, Johnson CA, et al. The Ocular Hypertension Treatment Study: baseline factors that predict the onset of primary open-angle glaucoma. Arch Ophthalmol. 2002; 120: 714-20- discussion 829-30. PMID: 12049575

3. Anderson DR, Drance SM, Schulzer M, Collaborative Normal-Tension Glaucoma Study Group. Natural history of normal-tension glaucoma. Ophthalmology. 2001; 108: 247-253. PMID: 11158794

4. Writing Committee for the Normal Tension Glaucoma Genetic Study Group of Japan Glaucoma Society, Meguro A, Inoko H, Ota M, Mizuki N, Bahram S. Genome-wide association study of normal tension glaucoma: common variants in SRBD1 and ELOVL5 contribute to disease susceptibility. Ophthalmology. 2010; 117: 1331-8.e5. doi: 10.1016/j.ophtha.2009.12.001 PMID: 20363506

5. Shibuya E, Meguro A, Ota M, Kashiwagi K, Mabuchi F, lijima H, et al. Association of Toll-like receptor 4 gene polymorphisms with normal tension glaucoma. Invest Ophthalmol Vis Sci. Association for Research in Vision and Ophthalmology; 2008; 49: 4453-4457. doi: 10.1167/iovs.07-1575

6. Burdon KP, MacGregor S, Hewitt AW, Sharma S, Chidlow G, Mills RA, et al. Genome-wide association study identifies susceptibility loci for open angle glaucoma at TMCO1 and CDKN2B-AS1. Nat Genet. 2011; 43: 574-578. doi: 10.1038/ng.824 PMID: 21532571

7. Wiggs JL, Yaspan BL, Hauser MA, Kang JH, Allingham RR, Olson LM, et al. Common variants at 9p21 and 8q22 are associated with increased susceptibility to optic nerve degeneration in glaucoma. PLoS Genet. 2012; 8: e1002654. doi: 10.1371/journal.pgen.1002654 PMID: 22570617

8. Fingert JH. Primary open-angle glaucoma genes. Eye. 2011; 25: 587-595. doi: 10.1038/eye.2011.97 PMID: 21562585

9. Rezaie T, Child A, Hitchings R, Brice G, Miller L, Coca-Prados M, et al. Adult-onset primary open-angle glaucoma caused by mutations in optineurin. Science. American Association for the Advancement of Science; 2002; 295: 1077-1079. doi: 10.1126/science.1066901

10. Alward WLM, Kwon YH, Kawase K, Craig JE, Hayreh SS, Johnson AT, et al. Evaluation of optineurin sequence variations in 1,048 patients with open-angle glaucoma. Am J Ophthalmol. 2003; 136: 904910. PMID: 14597044

11. Hauser MA, Sena DF, Flor J, Walter J, Auguste J, LaRocque-Abramson K, et al. Distribution of optineurin sequence variations in an ethnically diverse population of low-tension glaucoma patients from the United States. J Glaucoma. 2006; 15: 358-363. PMID: 16988596

12. Fingert JH, Robin AL, Roos Ben R, Davis LK, Scheetz TE, Wassink TH, et al. Copy number variations on chromosome 12q14 in patients with normal tension glaucoma. Hum Mol Genet. Oxford University Press; 2011; 20: 2482-2494. doi: 10.1093/hmg/ddr123

13. Kawase K, Allingham RR, Meguro A, Mizuki N, Roos B, Solivan-Timpe FM, et al. Confirmation of TBK1 duplication in normal tension glaucoma. Exp Eye Res. 2012; 96: 178-180. doi: 10.1016/j.exer.2011.12. 021 PMID: 22306015

14. Ritch R, Darbro B, menon G, Khanna CL, Solivan-Timpe F, Roos BR, et al. TBK1 Gene Duplication and Normal-Tension Glaucoma. JAMA ophthalmology. American Medical Association; 2014; 132 544-548. doi: 10.1001/jamaophthalmol.2014.104 
15. Liu Y, Garrett ME, Yaspan BL, Bailey JC, Loomis SJ, Brilliant M, et al. DNA copy number variants of known glaucoma genes in relation to primary open-angle glaucoma. Invest Ophthalmol Vis Sci. 2014; 55: 8251-8258. doi: 10.1167/iovs.14-15712 PMID: 25414181

16. Awadalla MS, Fingert JH, Roos BE, Chen S, Holmes R, Galanopolous A, et al. Copy Number Variations of TBK1 in Australian Patients With Primary Open-Angle Glaucoma. Am J Ophthalmol. 2015; 159: 124-130.e1. doi: 10.1016/j.ajo.2014.09.044 PMID: 25284765

17. Autophagy in the pathogenesis of disease. 2008; 132: 27-42. doi: 10.1016/j.cell.2007.12.018

18. Wild P, Farhan H, McEwan DG, Wagner S, Rogov VV, Brady NR, et al. Phosphorylation of the autophagy receptor optineurin restricts Salmonella growth. Science. American Association for the Advancement of Science; 2011; 333: 228-233. doi: 10.1126/science.1205405

19. Levine B. Eating oneself and uninvited guests: autophagy-related pathways in cellular defense. Cell. 2005; 120: 159-162. doi: 10.1016/j.cell.2005.01.005 PMID: 15680321

20. Wong E, Cuervo AM. Autophagy gone awry in neurodegenerative diseases. Nat Neurosci. 2010; 13: 805-811. doi: 10.1038/nn.2575 PMID: 20581817

21. Wong YC, Holzbaur ELF. Optineurin is an autophagy receptor for damaged mitochondria in parkinmediated mitophagy that is disrupted by an ALS-linked mutation. Proceedings of the National Academy of Sciences. National Acad Sciences; 2014; 111: E4439-48. doi: 10.1073/pnas.1405752111

22. Wong YC, Holzbaur ELF. Autophagosome dynamics in neurodegeneration at a glance. J Cell Sci. The Company of Biologists Ltd; 2015; 128: 1259-1267. doi: 10.1242/jcs.161216

23. Nixon RA. The role of autophagy in neurodegenerative disease. Nat Med. 2013; 19: 983-997. doi: 10. 1038/nm.3232 PMID: 23921753

24. Nassif M, Hetz C. Targeting autophagy in ALS: a complex mission. Autophagy. 2011; 7: 450-453. PMID: 21252621

25. Park HYL, Kim JH, Park CK. Activation of autophagy induces retinal ganglion cell death in a chronic hypertensive glaucoma model. Cell Death Dis. 2012; 3: e290. doi: 10.1038/cddis.2012.26 PMID: 22476098

26. Piras A, Gianetto D, Conte D, Bosone A, Vercelli A. Activation of autophagy in a rat model of retinal ischemia following high intraocular pressure. PLoS ONE. 2011; 6: e22514. doi: 10.1371/journal.pone. 0022514 PMID: 21799881

27. Morton S, Peggie M, Cohen P. Enhanced binding of TBK1 by an optineurin mutant that causes a familial form of primary open angle glaucoma. FEBS Lett. 2008; 582: 997-1002. doi: 10.1016/j.febslet.2008. 02.047 PMID: 18307994

28. Tucker BA, Solivan-Timpe F, Roos BR, Anfinson KR, Robin AL, Wiley LA, et al. Duplication of TBK1 Stimulates Autophagy in iPSC-derived Retinal Cells from a Patient with Normal Tension Glaucoma. Journal of Stem cell research \& therapy. 2014; 3: 161. doi: 10.4172/2157-7633.1000161

29. Pilli M, Arko-Mensah J, Ponpuak M, Roberts E, Master S, Mandell MA, et al. TBK-1 promotes autophagy-mediated antimicrobial defense by controlling autophagosome maturation. Immunity. 2012; 37: 223-234. doi: 10.1016/j.immuni.2012.04.015 PMID: 22921120

30. Cirulli ET, Lasseigne BN, Petrovski S, Sapp PC, Dion PA, Leblond CS, et al. Exome sequencing in amyotrophic lateral sclerosis identifies risk genes and pathways. Science. American Association for the Advancement of Science; 2015; 347: 1436-1441. doi: 10.1126/science.aaa3650

31. Freischmidt A, Wieland T, Richter B, Ruf W, Schaeffer V, Müller K, et al. Haploinsufficiency of TBK1 causes familial ALS and fronto-temporal dementia. Nat Neurosci. 2015; 18: 631-636. doi: 10.1038/nn. 4000 PMID: 25803835

32. Maruyama $\mathrm{H}$, Morino $\mathrm{H}$, Ito $\mathrm{H}$, Izumi $\mathrm{Y}$, $\mathrm{Kato} \mathrm{H}$, Watanabe $\mathrm{Y}$, et al. Mutations of optineurin in amyotrophic lateral sclerosis. Nature. 2010; 465: 223-226. doi: 10.1038/nature08971 PMID: 20428114

33. Fecto F, Yan J, Vemula SP, Liu E, Yang Y, Chen W, et al. SQSTM1 mutations in familial and sporadic amyotrophic lateral sclerosis. Arch Neurol. American Medical Association; 2011; 68: 1440-1446. doi: 10.1001/archneurol.2011.250

34. Rubino E, Rainero I, Chiò A, Rogaeva E, Galimberti D, Fenoglio P, et al. SQSTM1 mutations in frontotemporal lobar degeneration and amyotrophic lateral sclerosis. Neurology. Lippincott Williams \& Wilkins; 2012; 79: 1556-1562. doi: 10.1212/WNL.0b013e31826e25df

35. DeLuca AP, Whitmore SS, Barnes J, Sharma TP, Westfall TA, Scott CA, et al. Hypomorphic mutations in TRNT1 cause retinitis pigmentosa with erythrocytic microcytosis. Hum Mol Genet. Oxford University Press; 2016; 25: 44-56. doi: 10.1093/hmg/ddv446

36. Li H, Durbin R. Fast and accurate short read alignment with Burrows-Wheeler transform. Bioinformatics. Oxford University Press; 2009; 25: 1754-1760. doi: 10.1093/bioinformatics/btp324 
37. DePristo MA, Banks E, Poplin R, Garimella KV, Maguire JR, Hartl C, et al. A framework for variation discovery and genotyping using next-generation DNA sequencing data. Nat Genet. 2011; 43: 491-498. doi: 10.1038/ng.806 PMID: 21478889

38. Tucker BA, Scheetz TE, Mullins RF, DeLuca AP, Hoffmann JM, Johnston RM, et al. Exome sequencing and analysis of induced pluripotent stem cells identify the cilia-related gene male germ cell-associated kinase (MAK) as a cause of retinitis pigmentosa. Proceedings of the National Academy of Sciences. National Acad Sciences; 2011; 108: E569-76. doi: 10.1073/pnas.1108918108

39. Exome Aggregation Consortium (ExAC) [Internet]. 1st ed. Cambridge. Available: http://exac. broadinstitute.org

40. Hirano M, Nakamura $\mathrm{Y}$, Saigoh $\mathrm{K}$, Sakamoto $\mathrm{H}$, Ueno $\mathrm{S}$, Isono $\mathrm{C}$, et al. Mutations in the gene encoding p62 in Japanese patients with amyotrophic lateral sclerosis. Neurology. Lippincott Williams \& Wilkins; 2013; 80: 458-463. doi: 10.1212/WNL.0b013e31827f0fe5

41. Teyssou E, Takeda T, Lebon V, Boillée S, Doukouré B, Bataillon G, et al. Mutations in SQSTM1 encoding p62 in amyotrophic lateral sclerosis: genetics and neuropathology. Acta Neuropathol. Springer-Verlag; 2013; 125: 511-522. doi: 10.1007/s00401-013-1090-0

42. Shimizu H, Toyoshima $Y$, Shiga A, Yokoseki A, Arakawa K, Sekine Y, et al. Sporadic ALS with compound heterozygous mutations in the SQSTM1 gene. Acta Neuropathol. Springer Berlin Heidelberg; 2013; 126: 453-459. doi: 10.1007/s00401-013-1150-5

43. Le Ber I, Camuzat A, Guerreiro R, Bouya-Ahmed K, Bras J, Nicolas G, et al. SQSTM1 mutations in French patients with frontotemporal dementia or frontotemporal dementia with amyotrophic lateral sclerosis. JAMA Neurol. American Medical Association; 2013; 70: 1403-1410. doi: 10.1001/jamaneurol. 2013.3849

44. Kwok CT, Morris A, de Belleroche JS. Sequestosome-1 (SQSTM1) sequence variants in ALS cases in the UK: prevalence and coexistence of SQSTM1 mutations in ALS kindred with PDB. Eur J Hum Genet. 2014; 22: 492-496. doi: 10.1038/ejhg.2013.184 PMID: 23942205

45. Yang Y, Tang L, Zhang N, Pan L, Hadano S, Fan D. Six SQSTM1 mutations in a Chinese amyotrophic lateral sclerosis cohort. Amyotroph Lateral Scler Frontotemporal Degener. 2015; 16: 378-384. doi: 10. 3109/21678421.2015.1009466 PMID: 25708934 